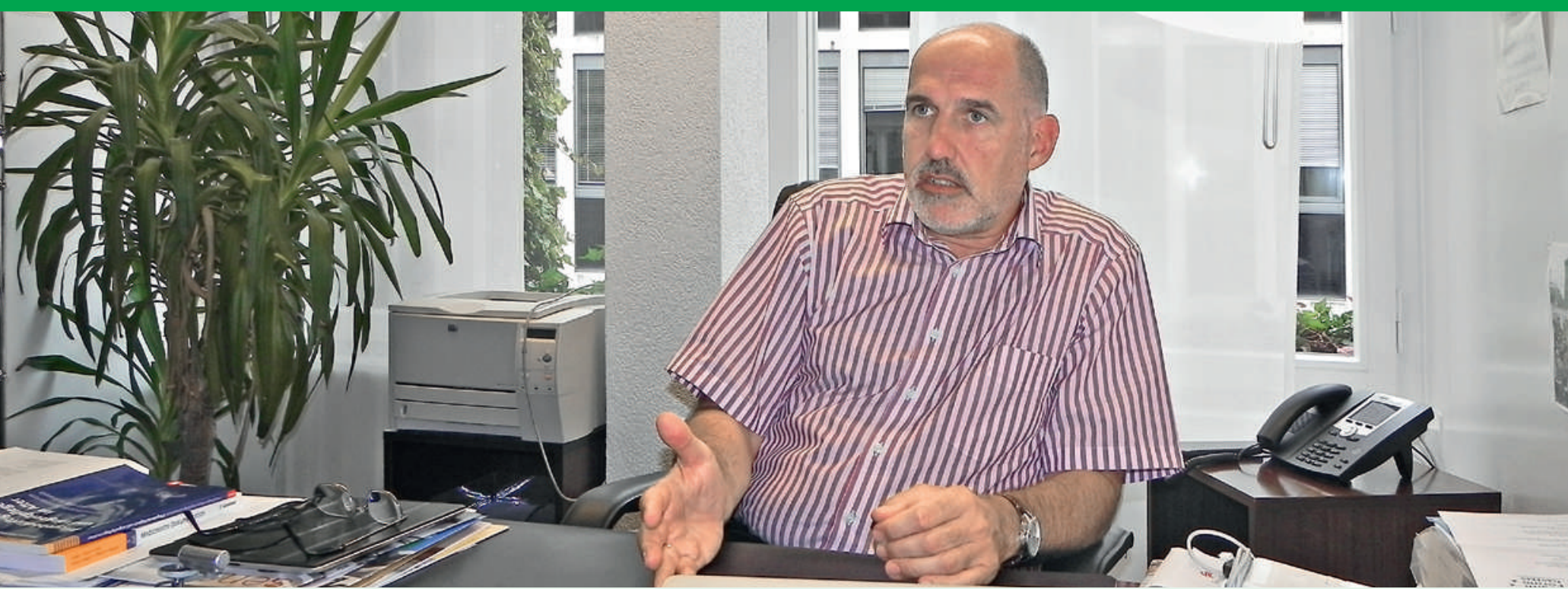

Während acht Jahren mit Leidenschaft Präsident der FMH: Jacques de Haller in seinem Büro an der Elfenstrasse in Bern.

\title{
«Die FMH ist immer noch da und stärker geeint als zuvor»
}

\author{
Während zweier Amtsperioden hat Jacques de Haller die Verbindung der Schweizer \\ Ärztinnen und Ärzte FMH an vorderster Front repräsentiert und deren Politik mass- \\ geblich mitgeprägt. Im Interview zieht der scheidende Präsident Bilanz, bevor er \\ die Amtsgeschäfte am 6. Dezember seinem Nachfolger Jürg Schlup* übergibt.
}

Interview: Bruno Kesseli

Der Abschied von Jacques de Haller ist auch Thema des Cartoons von ANNA auf der letzten Seite dieser Ausgabe.

\footnotetext{
* Der neue FMH-Präsident wird im Dezember in einem SÄZ-Interview vorgestellt.
}

Jacques de Haller, Sie haben sich während Ihrer achteinhalbjährigen Präsidialzeit mit voller Kraft und durchaus leidenschaftlich für die Standesorganisation eingesetzt und Sie hätten dies gern noch für eine weitere Amtsperiode getan. Dass es nicht geklappt hat, war sicher eine Enttäuschung. Mit welchen Gefühlen schauen Sie aus der Distanz von einigen Monaten auf die Ärztekammer vom vergangenen Juni zurück?

Jacques de Haller: Nicht wiedergewählt zu werden, war natürlich mit einer gewissen Enttäuschung verbunden - ein Drama war es aber nicht. Tatsache ist, dass ich lange überlegt habe, ob ich mich für eine dritte Amtszeit bewerben wollte. Angesichts der vielen für die Ärzteschaft wichtigen Fragen und Projekte, die anstanden, kam ich zum Schluss, dass eine weitere Amtsperiode Sinn machen würde. Ich habe den Job acht Jahre gemacht, und wenn ich auf diese Jahre zurückschaue, habe ich den Eindruck, ihn gut gemacht zu haben, mit positiven Resultaten für die Ärzteschaft. Die Ärztekammer hat entschieden. Dass es so herauskommen würde, zeichnete sich bereits im Vorfeld ab, war also keine Überraschung.

Warum haben Sie trotz der offenbar vorhandenen Warnsignale an Ihrer Kandidatur festgehalten?
Es gab eine beträchtliche Anzahl von Kolleginnen und Kollegen, die mich aktiv unterstützt haben. Ihnen gegenüber hätte ich es nicht in Ordnung gefunden, die Übung einfach abzubrechen und nicht mehr zu kandidieren. Zudem standen grosse politische Geschäfte an, die eine Kontinuität ebenfalls als sinnvoll erscheinen liessen. Ich erinnere daran, dass 10 Tage nach der Ärztekammer die Referendumsabstimmung zur Managed-Care-Vorlage mit dem überwältigenden Mehr von 76\% der abgegebenen Stimmen gewonnen wurde.

Ihr politisches Engagement und ihre Nationalratskandidatur für die SP hatten im Vorjahr innerhalb der FMH hohe Wellen geworfen. War diese Vorgeschichte im Hinblick auf Ihre Wiederwahl ein entscheidendes Handicap? Ich glaube es nicht. Mein Engagement für die SP hat tatsächlich zu einigen Reaktionen geführt, nachdem die Medien auf das Thema eingestiegen sind. In den Augen einiger Mitglieder der Ärztekammer wäre aber vor allem meine Tätigkeit im eidgenössischen Parlament problematisch gewesen ... und zu diesem Problem ist es bekanntlich nicht gekommen! Zudem haben in den letzten Jahren mehrheitlich die linken Parteien die Positionen der FMH mitgetragen. Meh- 
rere Mitglieder des neu gewählten Zentralvorstands gehören bekanntermassen der FDP an - sie werden vor der Herausforderung stehen, auch Parteien anderer politische Couleur für die Anliegen der FMH zu gewinnen. Und gleichzeitig das vielfältige politische Spektrum der Ärzteschaft gegen aussen zu tragen.

\section{«Ich habe den Job acht Jahre gemacht, und wenn ich auf diese Jahre zurückschaue, habe ich den Eindruck, ihn gut gemacht zu haben.»}

Die Kontinuität, die Sie sich für die FMH gewünscht haben, wird es nun wohl nicht geben ...

Ja, es wird anders sein, und die Ärztekammer hat es so gewollt. Ich sehe aber nach wie vor ein grosses Entwicklungspotential, und es wäre schade, wenn die Politik und der neue Stil der FMH es nicht erlauben würden, dieses Potential zu nutzen.

\section{Wie meinen Sie das?}

Die Entwicklung in den letzten Jahren war eindeutig, dass wir zum Hauptplayer in der Gesundheitspolitik geworden sind. Langfristig ist es für FMH und Ärzteschaft aus meiner Sicht entscheidend, die gesamte Gesundheitspolitik des Bundes mitzuprägen. Diesbezüglich haben wir uns in eine hervorragende Position gebracht. Wenn wir das aus den Augen verlieren und uns nicht mehr auf breite Perspektiven, sondern nur noch auf kurzfristige Ziele beschränken, werden wir wieder zum Spielball der Politik. Wobei ich nicht falsch verstanden werden möchte: Es gibt wichtige kurzfristige Ziele wie den Tarif. Es gibt aber andere Ebenen, die für die Zukunft der Ärzteschaft in der Gesellschaft sicher noch wesentlicher sind, und diese dürfen jetzt nicht vergessen gehen.

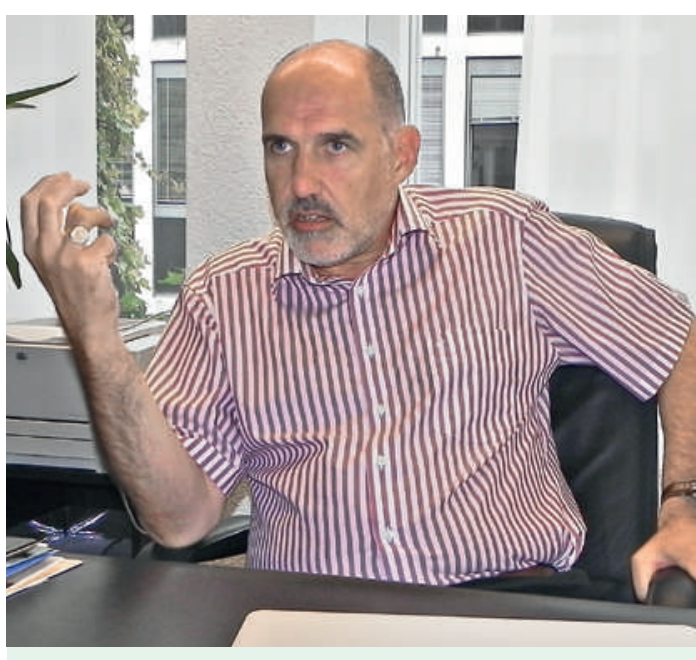

Jacques de Haller zieht eine positive Bilanz: «Die Entwick lung in den letzten Jahren war eindeutig, dass wir zum Hauptplayer im Gesundheitswesen geworden sind.»
Als Präsident der FMH war es Ihre Aufgabe, in wichtigen gesundheitspolitischen Fragen einen gemeinsamen Nenner der Ärzteschaft zu finden und diesen dann mit Überzeugung gegen aussen zu vertreten. Würden Sie nach acht Jahren sagen, dass diese Amtsauffassung an der Realität gemessen zu idealistisch ist?

Es ist wesentlich, dass die Ärzteschaft innerhalb der FMH zusammenbleibt. Wenn jede Untergruppierung ihren eigenen Kampf führt, für den Tarif oder für was auch immer, dann verlieren wir sehr viel Einfluss und Gestaltungsmöglichkeiten. Gegenstand der Politik zu werden und nicht mehr Subjekt zu sein, ist sicher nicht das, was ich mir für die FMH wünsche. Es braucht eine gewisse Einheit, einen gewissen Zentralismus. Man muss eine Vision haben, man muss wissen, was man will, man muss die Dinge - auch sich selbst gegenüber - begründen können.

Denken Sie, dass die gemeinsamen Interessen die Zentrifugaltendenzen auch auf längere Sicht ausgleichen können? Hat die FMH langfristig eine Zukunft?

Die Diskussion um Spaltungstendenzen ist nicht neu - bei meinem Amtsantritt gab es beispielsweise eine Krise mit den Chirurgen. Acht Jahre später stelle ich aber fest: Die FMH ist immer noch da und stärker geeint als zuvor. Wir haben die Kontroverse um die Integrierte Versorgung überstanden. Die Chirurgen sind geblieben, die Grundversorger sind geblieben, der VSAO ist geblieben. Wir haben jetzt fast 36000 Mitglieder, die gemeinsame Nenner haben.

\section{Als da wären?}

Die therapeutische Freiheit, das Anstreben der bestmöglichen Qualität, die freie Arztwahl, die Überzeugung, dass der Patient, die Patientin, im Zentrum des Gesundheitssystems stehen muss. Ja, die FMH hat eine Zukunft - wenn wir uns den zweifellos grossen Herausforderungen stellen.

\section{Zum Beispiel?}

Als Beispiel könnte ich hier die Grundversorgung nennen. Wir brauchen mehrere hundert Millionen Franken für die Besserstellung der Grundversorger, die wir sicher nicht bei einigen Spezialisten finden werden. Das Problem der Mehrkosten für die Grundversorger könnte hoffentlich über den Masterplan gelöst werden. Wenn aber am Ende die Grundversorger keine echte Unterstützung der FMH erhalten, werden sie Mühe haben, sich länger in der FMH zu Hause zu fühlen.

Bei der Abgabe eines Amtes liegt es nahe, Bilanz zu ziehen. In diesem Sinn möchten ich Ihnen einige Stichwörter zu wichtigen Themen Ihrer Amtszeit vorgeben und Sie bitten, diese kurz zu kommentieren. Als Erstes sei die Strukturreform der FMH angesprochen.

Die Anpassung der Strukturen mit der Schaffung der Delegiertenversammlung (DV) ist eine Erfolgsgeschichte. Wir erreichen dank dieser Institution 
konkrete Resultate, und das ist sehr gut für die FMH. Da jeder Dachverband eine Anzahl Delegierter stellt, die nicht direkt mit seiner Grösse verknüpft ist, ist eine Übervertretung einzelner Gruppen im Gegensatz zur Ärztekammer nicht möglich.

\section{«Es braucht eine gewisse Einheit, einen gewissen Zentralismus. Man muss eine Vision haben, man muss wissen, was man will.»}

Sprechen Sie hier die «Kickbacks» durch Spezialisten an, die unlängst ein Thema in den Medien waren?

Das ist ein Beispiel, ja. Kickbacks, auch von der Pharma, bedeuten Interessenkonflikte, über die die Patienten nicht informiert sind. Wenn Kickbacks möglich sind, stellt sich für den kritischen Beobachter aber auch die Frage, ob die Tarife der Spezialisten allenfalls zu hoch sind und gesenkt werden könnten. Es ist wichtig, dass die Ärzteschaft in heiklen Fragen dieser Art glaubwürdig bleibt.

\section{Nächstes Stichwort: TARMED.}

Der TARMED ist zu einer Normalität geworden. Er funktioniert, auch wenn er Anpassungen braucht. Die laufende Revision ist nicht einfach, weil sich die Ziele allmählich geändert haben. Aus meiner Sicht ist es vordringlich, zunächst die Situation für die Grundversorger zu verbessern und danach die anderen Probleme anzugehen. Wenn wir alles auf einmal lösen wollen, werden die Grundversorger nichts erhalten und die anderen ganz bestimmt auch nicht.

\section{Wie sehen Sie die Frage der Kostenneutralität?}

Es gibt von verschiedenen Seiten Bestrebungen, die Idee einer Kostenneutralität aufzugeben, damit eine Aktualisierung des Einkommens möglich wird. Eine finanzielle Besserstellung via Revision des TARMED und im Rahmen von TARVISION war ursprünglich nicht geplant. Wenn wir in diese Richtung gehen wollen, müssen wir das überzeugend begründen können, was nicht einfach wird.

\section{«Netzwerke und Integrierte Versorgung sind grundsätzlich eine gute Sache. Die Netzwerke müssen aber sehr darauf achten, dass sie ethisch absolut sauber funktionieren.»}

lichen auf Interventionen der Versicherer beim Parlament beruhten. Das führte dazu, dass die Vorlage von der FMH, der SP und der SVP abgelehnt wurde. Sorry, aber im letzten Moment mit «Tricks» zu kommen, ist einfach nicht akzeptabel und wurde auch vom Stimmvolk nicht goutiert. Wenn wir insgesamt mehr als drei Viertel der Stimmen erhalten haben, liegt dies sicher auch daran, dass wir Manöver dieser Art publik gemacht haben.

Wie sehen Sie die Situation der Integrierten Versorgung nach der Abstimmung?

Wie gesagt: Netzwerke und Integrierte Versorgung sind grundsätzlich eine gute Sache. Die Netzwerke müssen aber sehr darauf achten, dass sie ethisch absolut sauber funktionieren. Wenn kritische Fragen auftauchen und keine guten Antworten vorhanden sind, wird das problematisch.

\section{Stichwort DRG.}

Da ist die Situation in etwa ähnlich wie beim TARMED vor acht Jahren. Man kann aber sagen, dass das System an sich funktioniert, was auch für die SwissDRG AG gilt. Dass wir als FMH dort vertreten sind, ohne Tarifpartner zu sein, ist bemerkenswert und sicher von Vorteil. Wir müssen aber die Entwicklung des DRG-Systems sehr aufmerksam verfolgen, auch mit einer seriösen Begleitforschung. Nur so können wir frühzeitig reagieren, wenn Fehlentwicklungen oder gar Entgleisungen drohen.

\section{Stichwort Lobbying.}

Die Interessenvertretung im Bundeshaus und bei den Behörden ist für die Ärzteschaft zentral, und ich denke, wir haben in dieser Beziehung in den letzten Jahren sehr viel erreicht. Dabei habe ich von Anfang an sehr klar gemacht, dass für uns andere Kriterien gel- 
ten als für alle anderen Branchen, seien dies nun die Bauern, die Pharmaindustrie oder wer auch immer. Unser Tätigkeitsbereich betrifft zentrale Bereiche des Menschseins, es geht um Krankheit und Gesundheit, Lebensqualität, letztlich um Leben und Tod. Grössen wie Rentabilität, Effizienz, Gewinn oder Wettbewerb stehen dabei nicht an erster Stelle, sondern der Mensch - das haben wir beim Lobbyieren immer als Kernbotschaft kommuniziert.

\section{«Unser Tätigkeitsbereich betrifft zentrale Bereiche des Menschseins (...). Grössen wie Rentabilität, Effizienz, Gewinn oder Wettbewerb stehen dabei nicht an erster Stelle.»}

Wird eine solche Haltung von den «Partnern» in der heutigen «ökonomisierten Welt» ernst genommen?

Es brauchte viel Arbeit, bis diese Ideen im Parlament verstanden wurden, aber allmählich wurden sie akzeptiert. Ich kann jetzt in die Wandelhalle gehen und zum Beispiel sagen, «Sorry, diese Lösung ist zwar effizient, kommt aber für uns nicht in Frage, denn sie untergräbt das unabdingbare Vertrauensverhältnis zum Patienten». Diese Argumentationsweise wird mittlerweile bis hinauf zum Bundesrat verstanden. Das bedingt aber, dass wir absolut glaubwürdig sind, sonst sind wir bald der Lächerlichkeit preisgegeben.

\section{Stichwort Kontrahierungszwang.}

Die Debatte in diesem Bereich ist sehr ideologisiert. Grosse Parteien wie FDP und SVP streben nach wie vor die Aufhebung des Vertragszwangs an. Offensichtlich ist noch nicht allen klar, dass für die Bevölkerung die freie Arztwahl zentral ist: Darauf haben wir ja unsere Kampagne zur Managed-Care-Abstimmung ausgerichtet, und 76 Prozent der Bevölkerung stimmten zu.

\section{Stichwort Ärztemangel.}

Wir müssen Gesamtlösungen anstreben, da nicht nur die Ärzte, sondern die medizinischen Berufe insgesamt betroffen sind. Beim Nachwuchsproblem geht es nicht nur ums Geld - das Einkommen ist nicht mehr so gut wie früher, aber meistens immer noch anständig -, sondern es geht auch um Arbeitsbedingungen und Lebensqualität.

\section{Stichwort Medien.}

Wir müssen präsent sein, und auch in diesem Bereich wurde in den letzten Jahren ein grosser Schritt vorwärts gemacht. Wir sind jetzt Teil der Landschaft, man kann uns nicht einfach umgehen. Journalisten brauchen Informationen, um ihren Job machen $\mathrm{zu}$ können, und die können wir ihnen liefern. Im Allgemeinen funktionierte die Zusammenarbeit ausgezeichnet, unter über 100 Journalisten könnte ich vielleicht zwei, drei nennen, die ich als unanständig empfunden habe.

Wie erlebten Sie die Beziehung zu den wichtigen «Partnern» im Gesundheitswesen?

Lustigerweise hatten wir bis letztes Jahr eine «Westschweizer Exklusivität» im Gesundheitswesen: Der zuständige Bundesrat war Westschweizer, ebenso die Präsidenten von $\mathrm{H}+$, Santésuisse, pharmaSuisse, $\mathrm{GDK}^{*}$ und SBK${ }^{* *}$. Diese Konstellation ging mit einer guten Diskussionskultur einher, auch wenn wir in Sachfragen oft unterschiedlicher Meinung waren. An erster Stelle sind die Beziehungen aber immer personenabhängig.

\section{Können Sie konkrete Beispiele geben?}

Es ist uns beispielsweise nie gelungen, mit Bundesrat Couchepin ein konstruktives Gespräch zu führen. Machtfragen standen dort immer im Vordergrund. Mit Didier Burkhalter war die Zusammenarbeit dagegen gut, auch wenn seine Motivation nach einigen Monaten nachzulassen schien. Auch mit Alain Berset und seinem Team arbeiten wir ausgezeichnet zusammen. Ähnliches gilt für Santésuisse: Zum aktuellen Direktor von Santésuisse, Christoph Meyer, pflege ich eine sehr gute Beziehung.

Was sind Ihrer Ansicht nach die drängendsten Probleme und Fragen, mit denen sich die Ärzteschaft in der näheren und weiteren Zukunft konfrontiert sehen wird?

In der näheren Zukunft würde ich aus FMH-Sicht Hausarztinitiative und Einheitskasse nennen. Welche Position die FMH in der Frage der Einheitskasse beziehen wird, weiss ich nicht - ich finde es aber sehr

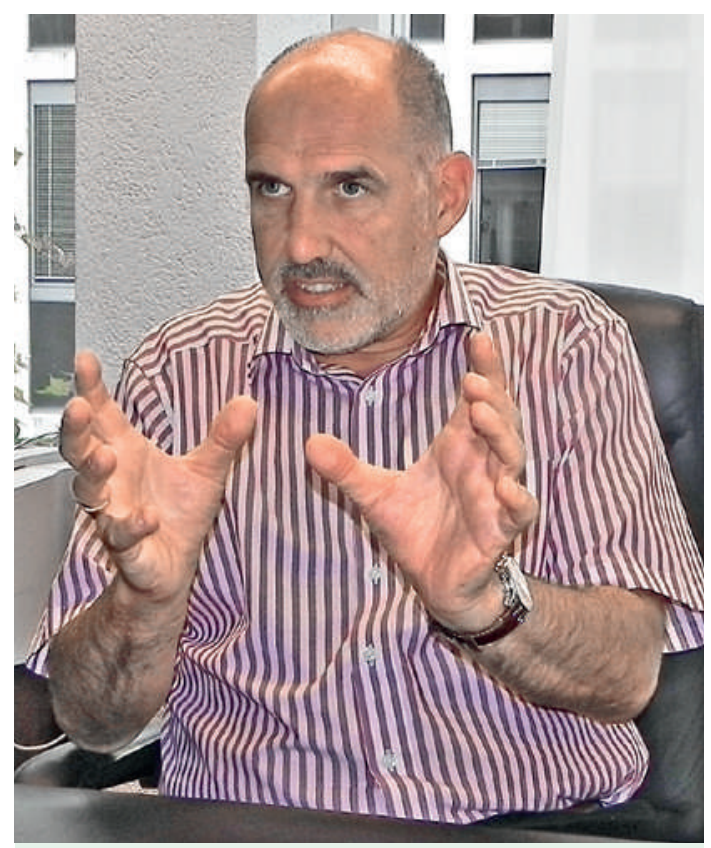

Aus der Sicht des scheidenden FMH-Präsidenten ist es wichtig, «unsere Vision einer guten Medizin» im Auge zu behalten. 


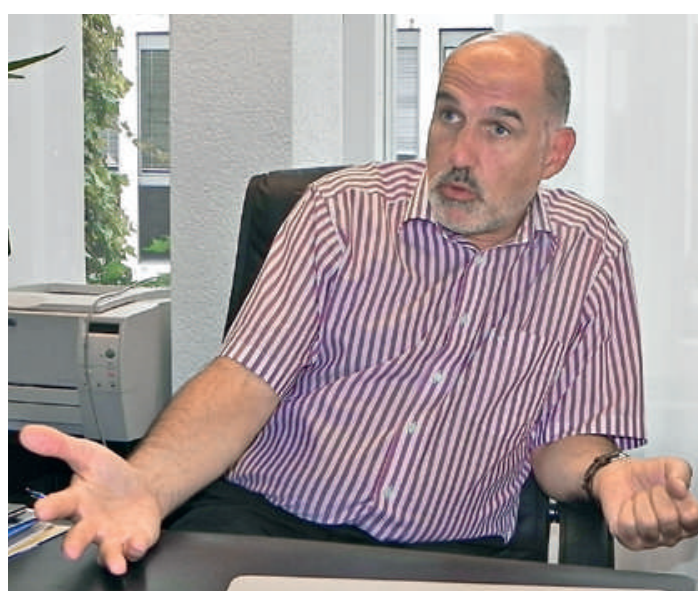

Keine Verschleisserscheinungen: Jacques de Haller fühlt sich heute fitter als vor seinem Amtsantritt.

wichtig, dass sich die Basis hier mit einer Urabstimmung ausdrücken kann. Sonst sind grosse Spannungen vorprogrammiert.

Wie sieht es in der längerfristigen Perspektive aus? Langfristig ist sicher die Finanzierung des Gesundheitswesens ein grosses Problem, wobei mir wichtig wäre, dass wir nicht nur unsere Tarife im Auge behalten, sondern unsere Vision einer guten Medizin. Diese breiteren Positionen müssen wir konsequent in die Politik einbringen, sonst finden wir uns bald am Rand und haben nichts mehr zu sagen.

Was meinen Sie mit «unsere Vision einer guten Medizin»? Ein Arzt hat jedes Jahr zwischen 2000 und 5000 Konsultationen, das sind in 20 Jahren bis 100000 Begegnungen. Dies führt zu einem unglaublichen Erfahrungsschatz und hoffentlich auch zu einem humanistisch geprägten Menschenbild, einer humanistischen Vision der Welt. Das alles nicht in die politisch-gesellschaftliche Diskussion einzubringen, sich nur auf das rein Medizinisch-Technische zu beschränken, wäre schade.

«Man muss ein solches Amt wollen und sich genau überlegt haben, was damit verbunden ist, weil es einen als Person sehr stark beansprucht.»

Damit haben Sie die Überleitung auf eine etwas persönlichere Ebene schon fast vollzogen. Welche Bereiche Ihrer Tätigkeit als Präsident der FMH haben Sie als besonders bereichernd erlebt?

Die Vielfältigkeit der Arbeit an sich ist natürlich eine Bereicherung: Dass das Spektrum so gross ist, wirkt immer wieder stimulierend. Sehr positiv erlebte ich auch die unzähligen persönlichen Kontakte mit ganz unterschiedlichen Menschen, den direkten Aus- tausch, auch die Überzeugungsarbeit, die zu leisten war.

Wie sieht es auf der Negativseite aus?

Als belastend empfunden habe ich vor allem die Vorurteile, mit denen ich immer wieder konfrontiert wurde und die häufig aus Kreisen standespolitischer Amtsträger kamen. Da gibt es politische Manöver, die primär Eigeninteressen und Machtansprüchen entspringen und sich innerhalb eines Verbands wie der FMH sehr lähmend auswirken. Wenn die FMH als Verband mehr Macht und Anerkennung erhält, wird das in diesen Kreisen als Bedrohung wahrgenommen und blockiert, um die eigenen Einflussbereiche zu sichern. Leider denke ich nicht, dass dies in den kommenden Jahren besser werden wird, aber die FMH wird damit klar kommen müssen.

Dass Ihr Amt mit einer hohen Belastung einhergeht, ist klar. Spüren Sie gewisse Verschleisserscheinungen?

Ich habe nicht das Gefühl, dass ich «verbraucht» bin. Man muss ein solches Amt wollen und sich genau überlegt haben, was damit verbunden ist, weil es einen als Person sehr stark beansprucht. Es ist politisch unkorrekt, aber eine Realität, dass ich nie eine echte Trennung zwischen Arbeit und Freizeit definiert habe. Das war einfach nicht möglich, aber dass ich mehr als 200\% gearbeitet habe, war für mich kein Problem. Die Arbeit als FMH-Präsident hat mich befriedigt, ich habe kein spezielles Hobby gebraucht. Wie gesagt mag das unkorrekt tönen, aber es ist so. Übrigens bin ich fitter als vor meinem Amtsantritt, mache zum Beispiel auch mehr Sport.

Wie werden spätere Generationen die «Ära Jacques de Haller» charakterisieren, welche Aspekte werden in Erinnerung bleiben?

Naturgemäss eine schwierige Frage. Aus heutiger Sicht würde ich die Positionierung der FMH in der Landespolitik nennen, die Tatsache, dass wir die effizienteste Lobby in der Schweiz geworden sind - diese Einschätzung stammt übrigens von einer sehr bekannten Zeitung. Das war sicher ein vorrangiges Ziel, das erreicht wurde, und das wird wahrscheinlich diese Periode am besten beschreiben.

Haben Sie schon Ideen, wie in welchen Bereichen Sie sich zukünftig engagieren möchten? Werden Sie dem Gesundheitswesen, der Gesundheitspolitik erhalten bleiben, oder möchten Sie sich neu orientieren und eine ganz andere Richtung einschlagen?

Die Richtung wird sicher im Bereich des Gesundheitswesens und der Gesundheitspolitik sein. Auf diesem Gebiet verfüge ich mittlerweile über grosse Erfahrung und ein gutes Netz. Es wäre schade, das einfach brachliegen zu lassen, zumal mich dieses Tätigkeitsfeld wirklich fasziniert. Was ich genau machen werde, ist zurzeit noch völlig offen. Aber das kommt, da mache ich mir keine Sorgen! 